\title{
Chin cup therapy for mandibular prognathism
}

\author{
Lee W. Graber, D.D.S., M.S., M.S. \\ Ann Arbor, Mich.
}

\begin{abstract}
$\mathrm{M}$
andibular prognathism has received the attention of dental clinicians for several hundred years. In 1778 , John Hunter, ${ }^{1}$ writing in his book The Natural History of the Human Teeth, stated: "It is not uncommon to find the lower jaw projecting too far forwards, so that its fore teeth pass before those of the upper jaw, when the mouth is shut; which is attended with inconvenience, and disfigures the face."

Goldstein, ${ }^{2}$ almost two centuries later, addressed the same problem, discussing its implications for modern orthodontic patients: "Mandibular prognathism or Class III malocclusion, has long been looked upon as one of the most severe facial deformities. Many patients afflicted with the condition have signified a willingness to face possible death rather than to continue to live thus deformed."

While the problem of mandibular prognathism has long been realized, a consensus as to its treatment has been lacking in the orthodontic literature. Perhaps part of the problem has been the infrequent appearance of the skeletal Class III malocelusion. Indeed, only about 5 per cent of the Caucasian population. is afflicted with skeletal mandibular prognathism. ${ }^{3-5}$ There has thus been a relative lack of pressure to confront this treatment problem; yet the patient with mandibular prognathism is of concern to the orthodontic practitioner, even if this patient constitutes only a small percentage of his practice.
\end{abstract}

From the Center for Human Growth and Development and Department of Anatomy, University of Michigan.

This article is taken in part from a thesis submitted in partial fulfillment of the requirements for the degree of Master of Science, 1)epartment of Orthodontics, Northwestern University. The research was supported in part by United States Public Health Service Research Grants 1722DE00193-01 and 1F32DE05011-01, National Institute of Dental Research, National Institutes of Health, Bethesda, Md.

Because of the extensive amount of tabular information in this study and publica. tion space limitations, additional tabular material may be secured by writing directly to the author. 
It was the purpose of this study to analyze cephalometrically a group of early mixed-dentition patients who had undergone 3 years of "chin cup" orthopedic therapy. In order to define the efficacy of the appliance, the treated sample was compared with a similar group of patients who did not receive treatment.

\section{Review of the literoture}

The use of restraining devices to reduce mandibular prognathism was reported in the early 1800's. ${ }^{6}$ Cellier in France and Fox, Kingsley, and Farrar in the United States all designed appliances that resemble today's chin cup. These early attempts to correct mandibular prognathism tended to fail for one of two reasons. First, the forces generated by appliances in the 1800 's were usually too small to have an influence on condylar growth mechanisms. ${ }^{7}$ Second, treatment was often begun after facial skeletal growth was completed, leaving the practitioner with the task of literally "driving" the mandible backward in the craniofacial complex. There was no clinical concept of growth guidance. The early failure with the chin cup appliance was one of the reasons that orthodontists turned to intraoral appliances with intermaxillary elastics in an attempt to correct the Class III problem. By their very nature, however, the intraoral appliances limited the clinician to a dental correction in a skeletal malocclusion. While it was sometimes possible to mask the skeletal disharmony between upper and lower jaws with a dental correction, the majority of cases that were treated were compromised and unsuccessful.

A conceptual change in the treatment of the Class III malocclusion was offered in the late 1940's and carly 1950 's. ${ }^{8}$ Aftcr obscrvation of the gross effects of Milwaukee brace treatment on the growth and form of the mandible, it was proposed that strong "orthopedic" forces in the range of 400 to $800 \mathrm{Gm}$. might be used to reduce a mandibular prognathism. The "orthopedic force" concept was put into actual use by directing strong forces to the mandibular basal bone through a chin cup mechanism. Although the design of the appliance was certainly not new, the use of heavy force was an important modification.

Several experimental animal studies have shown that force placed against the mandible can alter temporomandibular joint growth. ${ }^{9-14}$ However, parallel studies on human beings ${ }^{8,9,15-19}$ have not reported this finding. The human studies have had variable results because of differences in appliance design, force application, and evaluation methods. Those studies reporting failure have primarily been conducted on teen-age samples and have utilized comparatively light chin cup forces. Those studies reporting success, on the other hand, have emphasized the importance of early chin cup guidance with stronger orthopedic force. In short, chin cup orthopedic guidance of human mandibular development has been a subject for much opinion but little rescarch. Thus, the present study is an attempt to provide an experimental basis for chin cup therapy.

\section{Methods and material}

Sample. The material in this study consists of lateral cephalograms of thirty persons taken from the files of a private orthodontic practice. The treatment 

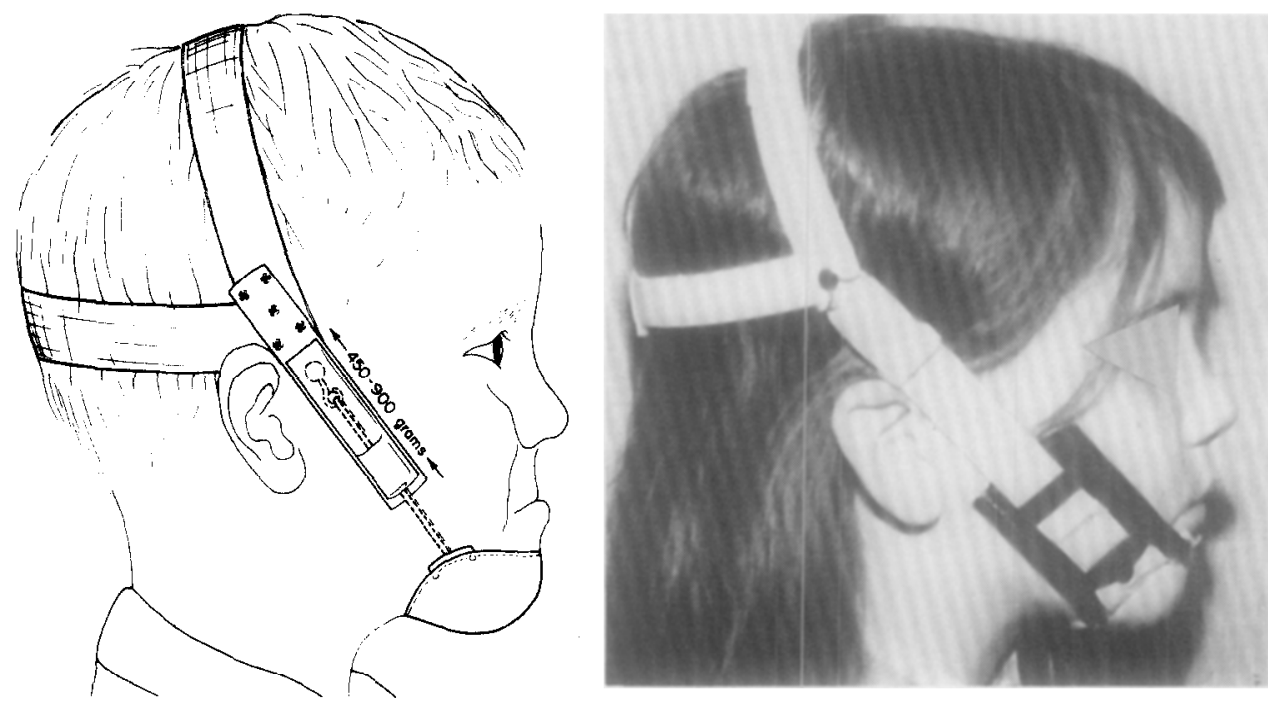

Fig. 1. The chin cup appliance is fabricated so that the pull on the chin cup is oriented along a line from the mandibular symphysis to the mandibular condyle. Maximum force levels ranged from 450 to $900 \mathrm{Gm}$. per side, depending on the developmental level of the patient.

group was selected for the purposes of this study according to the following criteria :

1. All subjects were from 5 to 8 years of age (average age, 6 years) at the start of treatment. This minimized the effect of any circumpubertal growth spurts during therapy.

2. No subject had undergone orthodontic therapy of any type prior to the start of this study.

3. No intraoral appliances were placed during the period of this study. This eriterion precluded any treatment effects other than those produced by the chin cup therapy.

4. In order to reduce skeletal variations and differing treatment responses that might be attributed to racial characteristics, all subjects were Caucasians.

5. All subjects in this group were under active chin eup guidance for a period of 3 years, (that is, from the beginning of the study to the final cephalometric film).

6. All subjects exhibited a skeletal Class III malocclusion prior to treatment. This was determined by clinical evaluation of each subject in centric relation in order to distinguish between skeletal and dental-functional Class III malocclusions. The mesiobuccal cusp of the maxillary first permanent molar (or deciduous second molar) occluded posterior to the buccal groove of the mandihular first permanent molar (or deciduous second molar). Moyers ${ }^{20}$ states that this ". . occlusal configuration almost always is the result of marked skeletal mandibular prognathism." 
Appliance. The appliance used on the treatment group was the "chin cup" appliance. This extraoral appliance utilizes a head cap which is firmly seated on the posterosuperior aspects of the cranium as anchorage and has attachments for the placement and activation of a chin cup. A force module of either elastic or metal coil spring was used to provide desired tension levels on the chin cup itself. The appliance was fabricated so that the pull on the chin cup was oriented along a line from the mandibular symphysis (pogonion) to the mandibular condyle (Fig. 1). While the materials used in the construction of this appliance varied slightly from patient to patient, the general design remained uniform.

Each patient began treatment with 150 to $300 \mathrm{Gm}$. of force per side of the chin cup. This force was increased to a minimum of $450 \mathrm{Gm}$. per side over a 2 -month period. Subjects were instructed to wear the chin cup for a period of 10 to 12 hours per day. In cases of more severe malocclusion or lack of cooperation, the period of requested wear was increased to 12 to 16 hours daily. After resolution of the skeletal maxillomandibular imbalance, both the amount of chin cup force and the duration of wear were reduced. The appliance was then used as a "retainer" for the remainder of the 3-year treatment period.

Treatment evaluation. Lateral cephalograms were taken at regular intervals during therapy, but for the purpose of this study only the beginning film and that film most closely approximating the completion of 3 years of active therapy were evaluated. Cartesian coordinates of thirty-three hard-and soft-tissue landmarks were recorded directly from the lateral cephalogram by means of a graph pen digitizer. Demographic material, including the subject's identification number, sex, age, birth date, film date, treatment type, and an index of patient cooperation was recorded with each film. A specially written Fortran IV computer program was used to generate the requested cephalometric measurements. There were fifty-eight variables in the study; the first variable, cooperation, was followed by fifty-seven linear and angular measurements (Fig. 2, $A-E$ ).

While most of the measures of this sample were based on classic methods and require little explanation, there is some variation in how the terms mandibular plane and occlusal plane are measured and interpreted. For the purposes of this study, the mandibular plane is defined as a line from menton to the point on the external contour of the posterior third of the mandible determined by a tangent through menton. The occlusal plane is synonymous with the "functional" occlusal plane and is defined by two points : the midinterocelusal point as measured at the mesiobuccal cusp of the maxillary first molar and the midinterocclusal point between the maxillary and mandibular first premolars (or, by default, the deciduous first molars). The use of the functional occlusal plane in this study was necessary to avoid variation in the plane due to incisor position.

Control group. Although the trealment group was a longitudinal sample, it was impossible to find longitudinal data on untreated skeletal Class III patients starting at 6 years of age. The young age of the group under study made even a cross-sectional analysis difficult. However, through the efforts of $\mathrm{G}$. Walker at the Biometries Laboratory of the University of Michigan School of Dentistry, an untreated, mixed longitudinal group analogous in age and skeletal characteristics was developed. 

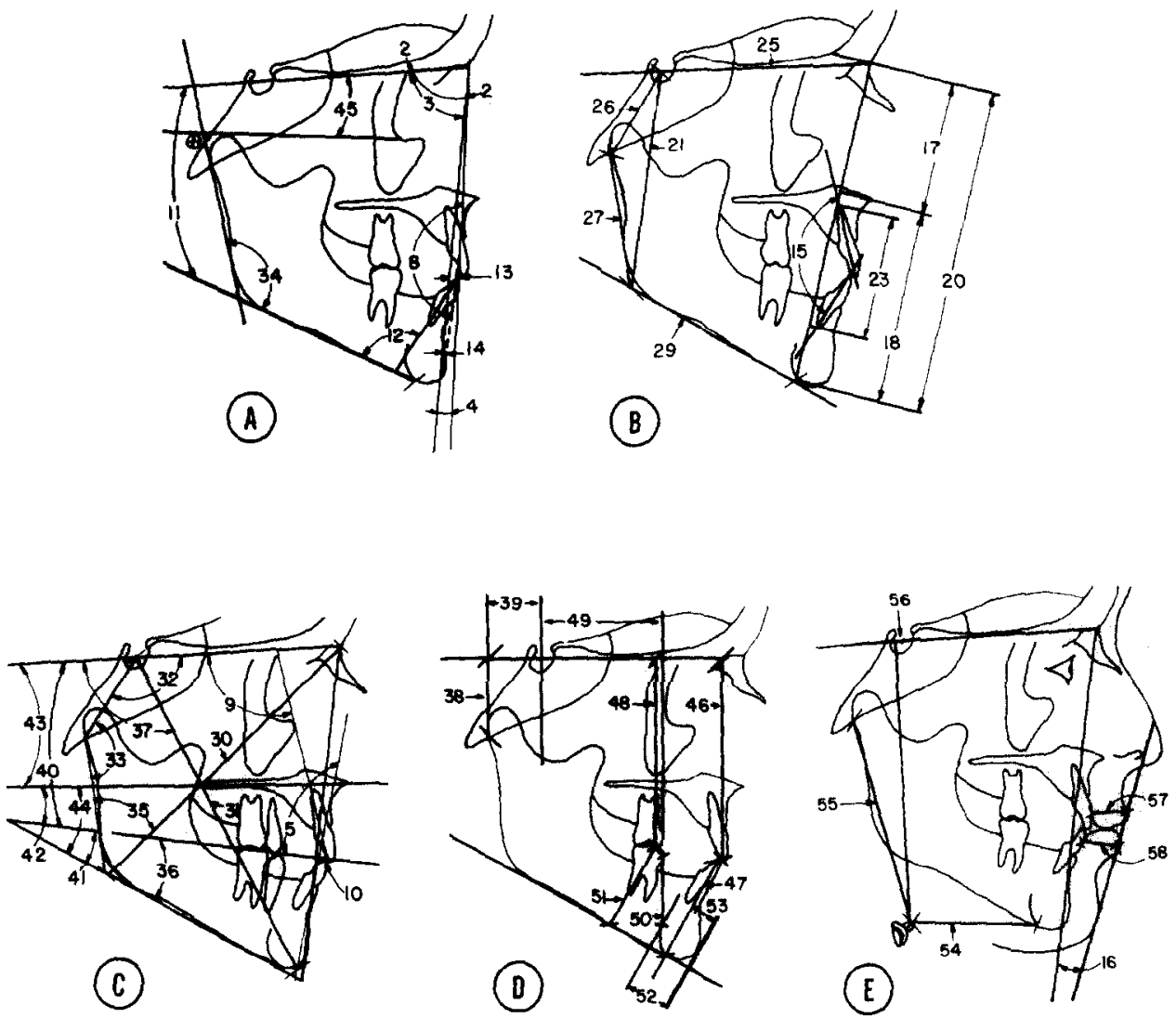

Fig. 2. A-E, Method of measurement. The number of the variable in the legend corresponds to the number code in the illustration. The first variable is cooperation. The others are: SNA (2); SNB (3); ANB (4); AB to occlusal plane (5); overjet (6); overbite (7); NAP (8); upper incisor to SN plane (9); upper incisor to facial plane (10); mandibular plane to SN plane (11); incisor-mandibular plane angle (12); lower incisor to NB (13); NB to pogonion (14); upper incisor to lower incisor (15); $\mathrm{H}$ angle (16); upper anterior face height (17); lower anterior face height (18); upper anterior face height to lower anterior face height (19); anterior face height (20); posterior face height (21); posterior to anterior face height (22); alveolar bone height (23); alveolar bone height to nasion-menton line (24); anterior cranial base length (25); posterior cranial base length (26); ramus height (27); posterior cranial base height to ramus height (28); mandibular body length (29); facial depth (30); facial length (31); saddle angle (32); articular angle (33); gonial angle (34); upper gonial angle (35); lower gonial angle (36); $Y$ axis (37); articulare to $S N$ vertical (38); articulare to $\mathrm{SN}$ horizontal (39); occlusal plane to $\mathrm{SN}$ plane (40); occlusal plane to mandibular plane (41); occlusal plane to palatal plane (42); palatal plane to SN plane (43); palatal plane to mandibular plane (44); Frankfort horizontal plane to SN plane (45); upper incisor incisal edge to SN plane (46); lower incisor incisal edge to mandibular plane (47); maxillary first permanent molar to SN vertical (48); maxillary first permanent molar to SN horizontal (49); mandibular first permanent molar to symphysis $(\mathbf{5 0})$; lower first permanent molar to mandibular plane vertical (51); symphyseal width (52); chin button size (53); hyoid to menton (54); hyoid to articulare (55); hyoid to SN plane (56); upper lip width (57); lower lip width (58). 
As only cephalometric values were available for this untreated group, the presence of a skeletal Class III malocclusion had to be defined in terms of cephalometric measurements rather than clinically. Walker ${ }^{21}$ stated that an ANB value of more than 1.5 standard deviations below the norm for the particular age in question is indicative of a Class III malocelusion as classically described in the literature. Using these criteria, sixteen subjects at age 6 , with ANB measurements of less than 1 degree, were chosen. Similarly, sixteen 9-year-old children with ANB angles of less than 0.5 degree were chosen. Walker ${ }^{21}$ analyzed forty-two variables in his sample and provided the incremental values of the differences in these variables for the 3-year period of growth used in this study.

Because the control group was selected on the basis of cephalometric parameters and was not examined clinically, an added evaluation of this sample was made to verify that the control group was, in fact, characteristic of Class III malocelusion. The initial age values of the untreated group were compared by paired $t$ tests to the initial values of the treatment groups (that is, a sample in which skeletal Class III malocclusion characteristics had been confirmed by both cephalometric and clinical examination). As skeletal Class III malocclusion involves discrepancies in a large number of areas within the craniofacial complex when compared to other craniofacial types, ${ }^{22}$ morphologie deviation by the control group from the known Class III skeletal pattern in the treated sample would be demonstrated by this statistical comparison. The results of this comparison indicated that the control sample was, indeed, representative of skeletal Class III malocelusion.

Statistical evaluation. The error of the method was studied by double-determination tracings and was evaluated statistically. This source of error was insignificant. Possible variations due to groupings by male and female sex were considered when compiling the samples for both the treatment and control groups. T tests were run on an initial set of variables in the group to note any differences due to sex. Of the fifty-seven variables considered, only eight were significant, and all of these were less than 0.05 . Because the variability between the male and female subgroups of the two samples was minimal, the sex factor was disregarded.

The nature of the control data required the utilization of mean variance scores for statistical computations, which tended to reduce the significance levels in the two comparison groups and biased the reported results in a conservative manner. This procedure thus minimized differences between the control and treatment groups that might be apparent with a more precise description of the control sample. However, because of this, results reported to be statistically significant in this study may be more readily accepted after a consideration of the conservative analytical bias present in this report.

Variations in the treatment time period were reduced to a period of 36 months for computation of the changes in variable values for both the treatment and control groups. Selection procedures (that is, selection of the treatment group on the basis of clinical analysis and selection of the control group on the basis of classic cephalometric values) did result in some differences between the two groups. However, the majority of these individual disparities were traced to 


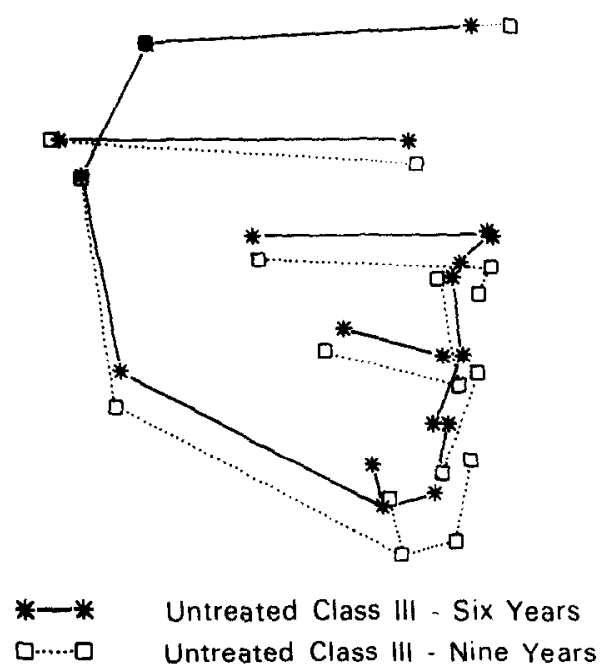

Fig. 3. This computer-composite tracing of a 9-year-old untreated Class 111 patient superimposed on a similar tracing of a 6 -year-old patient illustrates the continued dominance of mandibular prognathism after 3 years of growth.

differences in the cephalometric measurement techniques utilized in assessing the control and treatment groups. Comparisons between the two groups were thus made with respect to differences over the 3-year observation period rather than by variable-to-variable comparisons. Reducing the effect of errors inherent in the cephalometric technique permitted statistical comparisons (paired $t$ tests) to be made between the net changes noted in the 3-year observation period for both the treatment and control groups.

\section{Growth and treatment effects}

Study of the untreated sample indicated skeletal patterns and craniofacial growth trends similar to those reported by Björk, ${ }^{23}$ Joffe, ${ }^{24}$ and Rakosi. ${ }^{25}$ Subjects exhibited relative mandibular prognathism exemplified by poor mandibular position and excessive amounts of horizontal mandibular growth (Fig. 3). Horizontal growth excesses in the mandible were, for the most part, not matched by the maxilla, thus causing the Class III relationship to worsen with age. While growth discrepancies in the mandible and maxilla were the most obvious, there were deviations from normal skeletal form in the cranial base and in the positional relationship of the various craniofacial components. This finding supports the findings of Jacobson and co-workers, ${ }^{22}$ who stated that the "typical" Class III patient shows dysplasia to varying degrees throughout the eraniofacial complex.

The broad structural scope of the Class III skeletal problem was matched by the far-reaching effects of orthopedic force treatment (Table I). Comparison of variable changes over the 3-year period between the treated and untreated samples indicated that 3 years of chin cup therapy had ramifications in all areas of the craniofacial complex. While changes in some components were sometimes small, their compound effect resulted in an undeniable change in facial pattern 
Table I. Initial descriptive measures at 6 years of age and change after 36 months' treatment

\begin{tabular}{|c|c|c|c|c|}
\hline \multirow[b]{2}{*}{ Variable } & \multicolumn{2}{|c|}{6 years of age } & \multicolumn{2}{|c|}{ After 36 months' treatment } \\
\hline & Mean & $S . D$. & Mean & S.D. \\
\hline SNA (2) & $79.772 \mathrm{deg}$ & 2.4293 & $.17943 \mathrm{deg}$ & 1.2902 \\
\hline SNB (3) & $79.434 \mathrm{deg}$ & 2.5714 & $-1.3897 \mathrm{deg}$ & 1.8551 \\
\hline ANB (4) & $.33793 \mathrm{deg}$ & 1.6552 & $1.5820 \mathrm{deg}$ & 1.6266 \\
\hline $\mathrm{AB} / \mathrm{Occl}(5)$ & $82.190 \mathrm{deg}$ & 3.6855 & $3.4405 \mathrm{deg}$ & 3.7254 \\
\hline OJet (6) & $-.73448 \mathrm{~mm}$ & 1.2454 & $3.6152 \mathrm{~mm}$ & 2.0231 \\
\hline OBite (7) & $.74828 \mathrm{~mm}$ & 1.1485 & $1.3606 \mathrm{~mm}$ & 1.9324 \\
\hline NAP (8) & $1.8414 \mathrm{deg}$ & 3.7679 & $1.0304 \mathrm{deg}$ & 3.0692 \\
\hline UINS/SN (9) & $95.503 \mathrm{deg}$ & 10.765 & $8.2395 \mathrm{deg}$ & 9.4433 \\
\hline UINS/FP (10) & $1.9931 \mathrm{~mm}$ & 2.0385 & $2.5034 \mathrm{~mm}$ & 1.9560 \\
\hline MP/SN (11) & $34.334 \mathrm{deg}$ & 3.7831 & $-.74882 \mathrm{deg}$ & 1.9362 \\
\hline IMPA (12) & $85.131 \mathrm{deg}$ & 7.4004 & $2.5930 \mathrm{deg}$ & 6.3892 \\
\hline LINS/NB (13) & $1.8655 \mathrm{~mm}$ & 1.6025 & $.31628 \mathrm{~mm}$ & 1.7087 \\
\hline NB/POG (14) & $1.2586 \mathrm{~mm}$ & .84368 & $-.20068 \mathrm{~mm}$ & 1.2541 \\
\hline UINS/LOW (15) & $144.76 \mathrm{deg}$ & 13.588 & $-9.6106 \mathrm{deg}$ & 13.172 \\
\hline$H$ angle (16) & $9.1207 \mathrm{deg}$ & 4.1099 & $2.1482 \mathrm{deg}$ & 3.3772 \\
\hline U. Ant. face (17) & $43.086 \mathrm{~mm}$ & 2.9104 & $3.9791 \mathrm{~mm}$ & 1.7737 \\
\hline L. Ant. face (18) & $54.872 \mathrm{~mm}$ & 3.5757 & $3.3770 \mathrm{~mm}$ & 1.7581 \\
\hline UFace/LO(19) & 78.810 & 7.0870 & 2.3218 & 3.4762 \\
\hline Ant. face (20) & $97.910 \mathrm{~mm}$ & 4.8214 & $7.4165 \mathrm{~mm}$ & 2.7000 \\
\hline Post. fac (21) & $61.059 \mathrm{~mm}$ & 4.3251 & $5.9631 \mathrm{~mm}$ & 1.5315 \\
\hline $\mathrm{PO} / \mathrm{AN} F \Lambda(22)$ & 62.431 & 3.4049 & 1.3125 & 1.8325 \\
\hline Root I/I (23) & $27.714 \mathrm{~mm}$ & 5.5335 & $9.3466 \mathrm{~mm}$ & 5.6375 \\
\hline Alv./N-ME (24) & 28.252 & 5.0231 & 7.0324 & 5.5886 \\
\hline An. CRBSE (25) & $65.976 \mathrm{~mm}$ & 2.7823 & $2.5967 \mathrm{~mm}$ & 1.0007 \\
\hline PO CRBSE (26) & $28.845 \mathrm{~mm}$ & 2.5550 & $2.8342 \mathrm{~mm}$ & 1.2573 \\
\hline Ram. HT (27) & $35.855 \mathrm{~mm}$ & 3.0150 & $2.6245 \mathrm{~mm}$ & .1 .3654 \\
\hline PCB/RHT (28) & 80.703 & 6.6049 & 1.7774 & 5.0177 \\
\hline BOD length (29) & $58.500 \mathrm{~mm}$ & 3.7867 & $4.8291 \mathrm{~mm}$ & 2.1057 \\
\hline Face depth (30) & $98.017 \mathrm{~mm}$ & 4.3619 & $8.0381 \mathrm{~mm}$ & 2.3493 \\
\hline Face length (31) & $106.05 \mathrm{~mm}$ & 4.8943 & $6.6071 \mathrm{~mm}$ & 2.9782 \\
\hline SAD angle (32) & $121.38 \mathrm{deg}$ & 4.5808 & $.89716 \mathrm{deg}$ & 2.1697 \\
\hline ART angle (33) & $142.54 \mathrm{deg}$ & 6.0411 & $2.4520 \mathrm{deg}$ & 3.4683 \\
\hline GON angle (34) & $130.82 \mathrm{deg}$ & 4.7067 & $-5.1186 \mathrm{deg}$ & 2.4366 \\
\hline UGON angle (35) & $58.472 \mathrm{deg}$ & 3.6801 & $-4.0611 \mathrm{deg}$ & 2.1693 \\
\hline LGON angle (36) & $72.348 \mathrm{deg}$ & 2.9318 & $-1.1251 \mathrm{deg}$ & 1.8617 \\
\hline$Y$ axis $(37)$ & $117.64 \mathrm{deg}$ & 2.7146 & $-1.7418 \mathrm{deg}$ & 2.3055 \\
\hline $\mathrm{ART} / \mathrm{SN} / \mathrm{V}(38)$ & $24.324 \mathrm{~mm}$ & 2.2855 & $2.0721 \mathrm{~mm}$ & 1.5350 \\
\hline ART/SN/H (39) & $14.776 \mathrm{~mm}$ & 2.8056 & $2.2757 \mathrm{~mm}$ & 1.2121 \\
\hline $\mathrm{OP} / \mathrm{SN}(40)$ & $19.786 \mathrm{deg}$ & 2.7986 & $.41499 \mathrm{deg}$ & 3.3905 \\
\hline $\mathrm{OP} / \mathrm{MP}(4 \mathrm{l})$ & $14.659 \mathrm{deg}$ & 3.2601 & $-.94223 \mathrm{deg}$ & 3.3865 \\
\hline OP/PALPL (42) & $11.697 \mathrm{deg}$ & 3.4025 & $-.36772 \mathrm{deg}$ & 3.3342 \\
\hline PALPL/SN (43) & $7.8379 \mathrm{deg}$ & 2.4720 & $.89196 \mathrm{deg}$ & 2.2550 \\
\hline PALPL/MP (44) & $26.324 \mathrm{deg}$ & 3.6202 & $-1.1697 \mathrm{deg}$ & 2.6988 \\
\hline $\mathrm{FH} / \mathrm{SN}(45)$ & $7.0517 \mathrm{deg}$ & 3.5905 & $-.41881 \mathrm{deg}$ & 2.8842 \\
\hline
\end{tabular}

as a direct result of therapy (Fig. 4). The various areas of the face will be considered separately for purposes of discussion. However, the reader should keep in mind the additive and sometimes multiplicative treatment effect indicated by combining the individual area observations into the functional entirety of the craniofacial complex. 


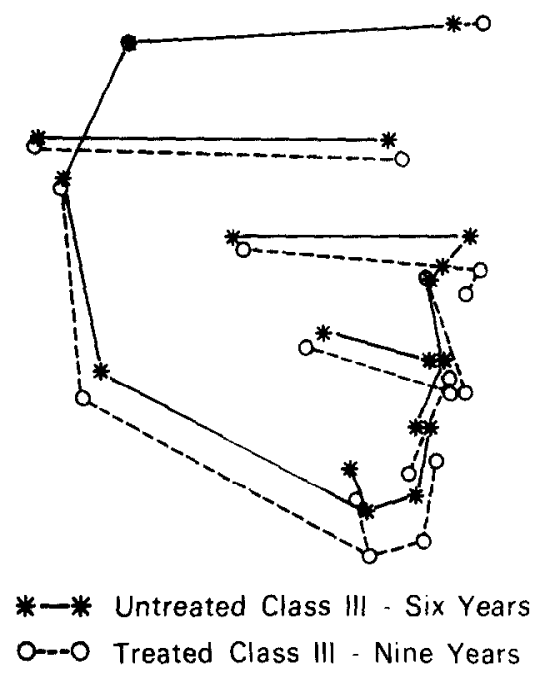

Fig. 4. This computer-composite superimposition of a 9-year-old Class III patient over the 6 -year-old Class 111 tracing illustrates the correction obtained after 3 years of orthopedic chin cup therapy.

Cranial base relations. The measurements of cranial base structures were divided into two categories-an evaluation of component part size and an evaluation of angular relationships. Analysis of anterior cranial base length revealed that the treated sample exhibited less growth in length than did the untreated sample. The disparity of more than $1.0 \mathrm{~mm}$. in this measure may be attributed to some retardation in the anteroposterior growth at the spheno-ethmoidal synchondrosis. It can be assumed that forces were transferred through the maxilla, by way of the teeth, from the mandible.

At the end of the treatment period the average length of the posterior cranial base was not significantly different for either the control or the treatment group. However, the relation of the anterior cranial base to the posterior cranial base (that is, the saddle angle) did change. The control sample showed an opening of this angle over the 3-year period of almost 5.5 degrees; the treatment group showed an opening of approximately 1.0 degree. While changes in both samples were statistically significant, one might have expected the opposite results, namely, an opening of the saddle angle induced by posterior orthopedic forces and a closing of the saddle angle as mandibular prognathism became more acute in the control sample. The reasons behind the noted differences are obscure. However, Henry ${ }^{26}$ has noted calvarial and "clockwise" cranial base changes after the use of heavy orthopedic forces in studies of Macaca mulatta monkeys. One may speculate that there were similar cranial base rotations in the human treated sample which, because of the lack of metallic cranial base implants, we were unable to measure.

Cranial base-mandibular relations. The control group showed an inerease in the SNB angle, while the treatment group showed a decrease. The backward positioning of point $\mathrm{B}$ was due in part to a backward rotation of the mandible.

Postural positioning of the mandible was assessed by evaluation of the articular angle (S-Ar-Go). While control subjects showed a closure of the 
articular angle, the patients receiving chin enp therapy showed an opening. This change in angular relationship between the ascending ramus and the cranial base tends to decrease the horizontal expression of the ramus length and to increase its vertical contribution relative to other craniofacial structures.

The measurements designed to explore alterations in the position of the temporomandibular articulation relative to the cranial base (Fig. $2, D$ ) indicated that the temporomandibular joint moved downward and backward with growth. In the control sample, it moved more posteriorly than inferiorly. The treatment group showed a slight predilection to posterior horizontal change over inferior vertical change, but any "orthopedic" effect appears to have been minimal.

Both control and treatment groups showed a decrease in the mandibular plane angle. However, the control group showed a greater change by almost three to one $(p<0.001)$. Part of this difference may be explained by the backward rotation of the mandible relative to the cranial base in the treatment sample. Horizontal growth vectors appear to have been more vertically directed during the treatment periad.

Mandibular relations. With respect to mandibular relations, analysis may be considered in terms of changes in localized mandibular contour and changes in the relationship of the component parts of the mandible. An obvious skeletal change that one would expect from the use of the chin cup appliance is alteration of bony contours at the mandibular symphysis. In our treated sample, however, measurements of the chin button showed no alteration in size. The entire symphysis did decrease slightly in width, but this change was not statistically significant. Apparently, the localized pressure effects of the chin cup itself were sufficiently dispersed so that no one area was impinged upon with a duration and intensity enough to cause bone remodeling.

Alterations in mandibular body length (gonion to menton) were monitored in both the treatment and control groups. The treatment group showed $1.0 \mathrm{~mm}$. less anteroposterior growth than did the control group, but this difference was not statistically significant.

The measure of posterior vertical mandibular body size showed significant differences between control and treatment samples. A smaller increase in height was secn in the treated sample, leading to the conclusion that there was some retardation of posterior vertical alveolar increase during the orthopedic treatment.

With respect to the relationship of the mandible to the cranial base, there was a change in the orientation of the ramus to the posterior cranial base; that is, posterior rotation of the mandible centered at the temporomandibular joint caused the ramus to be oriented in a more vertical position. This new angular relationship resulted in a more vertical expression of ranus size.

Evaluation of changes in length of the ascending ramus proved to be even more significant from the standpoint of altering growth. While both the treatment and control groups showed increase in ramus length with growth, the control sample had an average of more than $3.0 \mathrm{~mm}$. greater growth than the treatment group $(p<0.001)$. This suggests that the orthopedic chin cup effect 
was transmitted through the mandible, placing pressure on the condylar growth site and thus causing the observed diminution in ramus length. This study provides the first confirmative evidence of vertical condylar growth retardation in human beings.

The relationship of the mandibular body to the mandibular ramus, as expressed by the gonial angle, provided further evidence of orthopedic effect. While both control and treatment groups showed a decrease in this angle, the subjects treated with chin cup therapy showed almost 2 degrees more closure than the untreated group.

In describing the Jarabak-University of Chicago cephalometric analysis, $J^{J}$ arabak $^{27}$ emphasized the importance of the upper and lower components of the gonial angle and stated that lower gonial angle values of between 70 to 75 degrees are normal, while values of 75 degrees and above indicate excessive lower face height. Lower gonial angles (70 degrees and below) indicate diminished lower face height. In the treatment group reported in this study there was a statistically significant decrease to 71 degrees in this angle, giving the treatment group a relatively normal lower face height at the end of the treatment period.

The upper gonial angle measures have significance in predicting growth direction. ${ }^{27}$ If the value is above 55 degrees, the person tends to show a horizontal mandibular growth pattern. Normal values are between 50 and 55 degrees, with those subjects below 50 degrees tending to show a vertical mandibular growth pattern. In the treated subjects from this study, there was a decrease in gonial angle from 58.5 degrees to 54.4 degrees $(p<0.001)$. Thus, the treated sample was changed from a dominant horizontal mandibular growth pattern to one that fell within the range of normality.

Maxillomandibular relations. Changes in the ANB and NAP angles were monitored for both groups. In the untreated group, the ANB angle continued to indicate a worsening of the Class III skeletal maxillomandibular relationship. Those subjects treated with the chin cup, however, exhibited a normalization of the mandible relative to the maxilla. Similar findings in the NAP angle and in the $\mathrm{AB}$ to functional-occlusal plane relationship supported these observations. While the ANB angle does not indicate where changes were affected, it does mirror the resolution of the skeletal malocelusion that was seen elinically.

The angular relationship of the mandibular body to the palate was determined by using the relationship of the palatal plane to the mandibular plane angle. Both groups showed a closure of the angle, with the control group showing twice as much closure as the treated sample. Although these differences were not statistically significant, these findings do demonstrate a trend which is supportive of the rotational change in mandibular and palatal positions noted in the treatment group.

Maxillary relations. Evaluations of upper face changes over the 3-year period were made by measuring vertical height in the anterior and posterior maxilla. As was the case with the mandible, measures were taken from the occlusal surfaces of the molars and the incisors in order to test the total effective vertical change in the upper face (Fig. 2, D). A measure of the relationship of the palatal 
to occlusal planes showed no change in either the treatment or the control group, indicating that vertical changes observed through dental-cranial base measures reflected the vertical orientation of the maxilla to the basicranium.

For purposes of this study, analysis was divided into a consideration of both the anterior and posterior maxilla. Upper anterior face height was measured both directly (Fig. 2, $C$ and $D$ ) and indirectly (by measuring from the SN line to the tip of the maxillary central incisor). Both control and treatment groups showed vertical growth in the anterior face. While differences between the two groups were not statistically significant, the treated sample did show a slightly greater increase in anterior face height.

In the posterior face, however, differences between the control and treatment groups were statistically significant $(\mathrm{p}<0.01)$. The sample treated orthopedically showed an increase in vertical dimension which was one half that of the untreated sample. Actual vertical dimensional measures, when considered with an analysis of the palatal plane to SN line angle (see below) indicale that the orthopedic forces contributed to a decrease in the posterior maxillary vertical development. The parallel nature of the occlusal and palatal planes suggests action at either alveolar or combined alveolar and basal bone sites.

Cranial base-maxillary relations. Analysis indicated that while the angle defining the relation of the palatal plane to the SN line closed slightly in the control sample, it opened in the treated sample $(p<0.01)$. This indicates that the orthopedic forces of the chin cup mechanism were transferred through the posterior dentition to the posterior maxilla. Vertical growth was altered, producing a small but definite rotation of the maxilla that resulted in opening of the palatal plane relative to the $\mathrm{SN}$ angle.

Anteroposterior movement of the maxilla was measured by the SNA angle. There was negligible change and no difference between the treatment sample and the control sample. This, when combined with the findings on maxillary height, indicates that the orthopedic effects of the chin cup on the maxilla, as transferred through the mandible, were primarily vertical rather than horizontal.

Dental relations. While one might expect the effects of the chin cup to be localized primarily in the anterior segments of the dentition, with lingual inclination of the mandibular incisors, a comparison of treatment and control groups in this study proved just the opposite (Fig. 5). Indeed, while untreated subjects were characterized by an increasing lingual inclination of the lower incisors, the treated sample showed a relative labial inclination approaching commonly accepted normative values for IMPA. Thus, the localized dental effect in this study may be discounted as contributing significantly to the correction of the Class III malocclusion. A more plausible explanation is that the lower incisor inclination was responsive to the induced alterations in maxillomandibular relationships.

Changes in maxillary incisor position were significantly different between treatment and control groups. While the control group showed continued uprighting of the maxillary incisor, as measured by the upper incisor to the SN angle and the upper incisor to facial plane distance, the treatment group tended to normalize. The forward angulation and position of the maxillary incisors contributed to the reduction of the Class III dental malrelationship. However, 


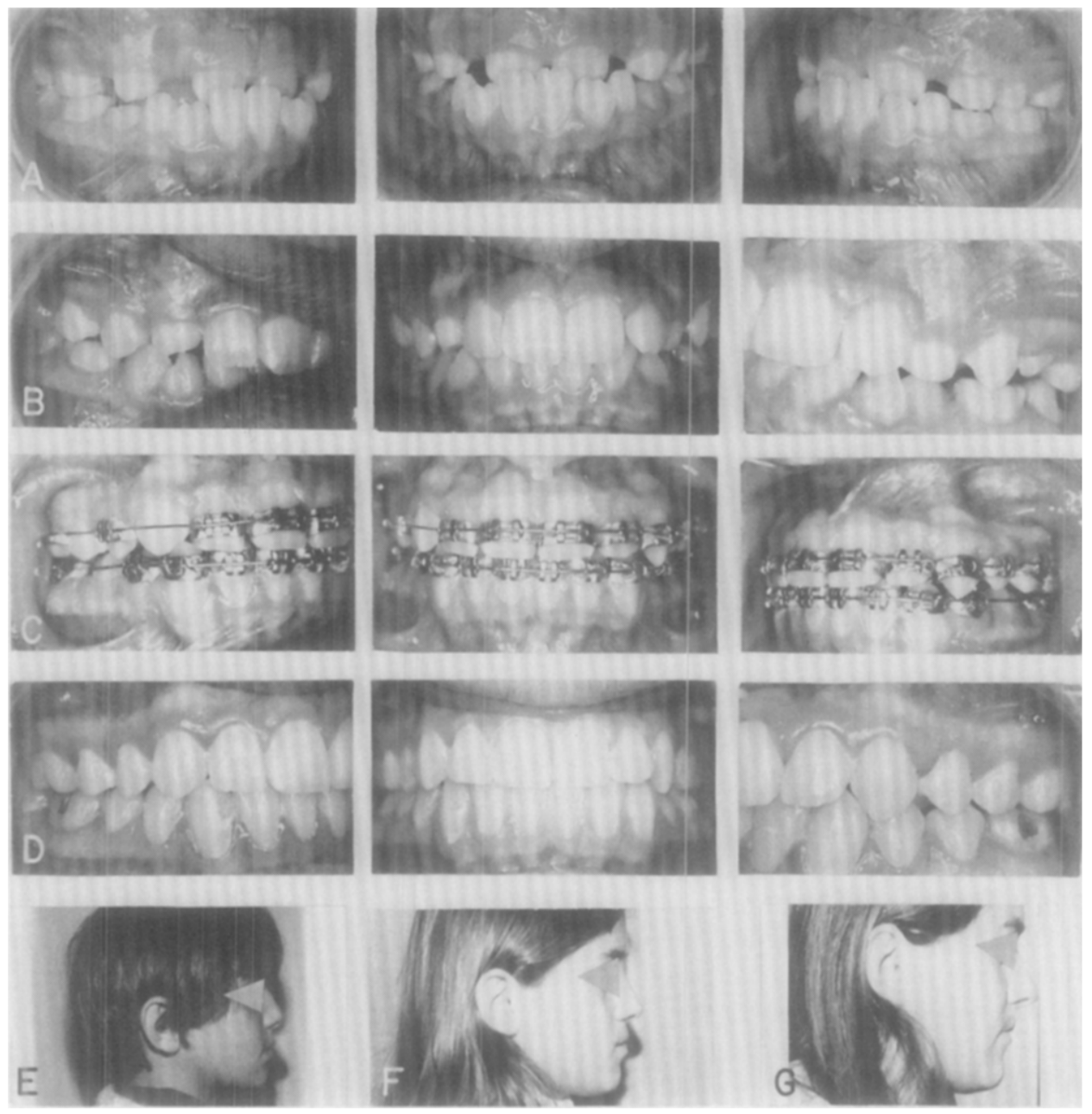

Fig. 5. Early orthadontic guidance cases require orthodontic supervision until the end of the circumpubertal skeletal growth phase. This photographic series illustrates that point for the chin cup patient. A, Patient with familial history of mandibular prognathism exhibits a Class III malocclusion at age 7. B, Photographs indicate normalization of maxillomandibular relations following $2 \frac{1}{2}$ years of chin cup guidance. C, Appliances placed to correct incisor alignment. Maxillomandibular relationship maintained with chin cup during circumpubertal growth spurts. D, Five years after all orthodontic appliances have been removed. E, Pretreatment profile at age 7. F, Post-chin cup therapy at age 10. G, Postretention profile at age 19.

maxillary incisor inclination at the end of the 3-year period was not excessive.

The relationship between the maxillary and mandibular incisors improved in the treatment group as a function of the incisor segment position and angular improvement of the individual tooth within the respective jaws. These changes are believed to be secondary to the alteration of the maxillomandibular relationship produced by the orthopedic chin cup. 
Statistically significant changes in overbite and overjet also reflected the relationship between the maxilla and the mandible. While the control group showed a worsening negative overjet and an increase in levels of overbite due to characteristic "overclosure," the treatment sample exhibited just the opposite changes; overjet improved almost $2.0 \mathrm{~mm}$. in the treated sample, while overbite increased $1.0 \mathrm{~mm}$., helping to stabilize the corrected skeletal dental relationship.

Soft-tissue relations. Soft-tissue changes were monitored by measuring the Holdaway " $\mathrm{H}$ " angle and upper and lower lip thickness. Lip thickness showed no appreciable change during the treatment period. However, there was an alteration in soft-tissue facial profile as indicated by the " $\mathrm{H}$ " angle $(\mathrm{p}<0.0016)$. This change indicated alteration from a prognathic outline to a straighter profile type. While angular values were on the "prognathic" side of normal for the age group in question, a considerable change from pretreatment values was reported. Unavailability of soft-tissue point recordings for the control group made intergroup comparison unfeasible.

Facial dimensional changes. There were obvious size increases in both anterior and posterior face height during growth, as reported above. The interesting points of comparison between treatment and control groups revolve around the relative contributions of the specific components that comprise the vertical changes in both anterior and posterior regions.

While the total anterior face height change over the 3-year period was similar for both groups, there was a difference in the way it was apportioned between the upper and lower segments. The control group maintained the ratio of upper to lower face fairly closely, with the lower face increasing slightly more than the upper face. In the treatment sample the situation was reversed, with the upper face showing more vertical increase than the lower face. This resulted in a statistically significant change in the ratio of the upper to the lower face.

The posterior face showed statistically significant $(\mathrm{p}<0.01)$ differences between the two groups, with the treated sample increasing less than the untreated sample. The locus for this difference appears to be in the retardation of growth in the mandibular condyles of the treated subjects. While the posterior cranial base height increase in both samples was about equal, there was a large difference in the ramus height growth, leading to the observed differences in total posterior face height.

The ratio of posterior to anterior face height indicates that anterior face height increased more rapidly, particularly in the treated sample. It is suggested that the orthopedic effect of the chin cup appliance was successful in retarding posterior vertical face growth to some degree and in slightly increasing anterior face height. The combined effect is observed as a downward and backward facial rotation. While this rotational change has not been previously reported in human. studies, Janzen and Bluher ${ }^{11}$ and Joho ${ }^{3}$ made similar observations on parallel studies in rhesus monkeys.

The importance of the relative position of the component parts of the face in contrast to singular alterations in size is again illustrated by the comparison of facial length (sella-gnathion) changes to alterations in the $\mathrm{Y}$ axis. While no significant difference was seen between the two groups in the size measure (facial 
length), there was a statistically significant difference $(\mathrm{p}<0.001)$ in the $\mathrm{Y}$ axis. Untreated skelctal Class III patients exhibited an opening of the axis, while orthopedically treated patients showed a closing of the axis (that is, a backward rotation). The closure of the $\mathrm{Y}$ axis may, in turn, be linked to a more vertical expression of facial growth, particularly mandibular growth.

\section{Role of cooperation}

The expected degree of patient cooperation was evaluated at the beginning, during, and at the end of the treatment period. A three-point grading system was used, with " 1 " indicating poor cooperation (approximately one half the requested appliance wear), "2" indicating good cooperation (almost all of the requested appliance wear), and " 3 " indicating excellent cooperation (better than the requested appliance wear). Both parents and treatment subjects were questioned concerning the amount of appliance wear, and all results were noted on the patient's records. Cooperation levels were recorded separately from treatment results.

There was little change in the level of cooperation, once a pattern was established. There was a very slight tendency in this sample toward decreased levels of cooperation with time, but the finding was not statistically significant. At the age level involved in this study, the subjects were generally very cooperative. Girls generally cooperated better than did boys.

The change in $\mathrm{AB}$ to occlusal plane angle was chosen as being representative of treatment success..$^{28}$ Large increases indicated suceess of treatment (that is, reduction of maxillo-mandibular malrelationship). Small changes indicated deficiencies in treatment. A strong positive correlation was found between treatment success and cooperation in appliance wear. In other words, those subjects who cooperated in wearing the chin cup appliance exhibited the greatcst amount of correction of their skeletal malocclusion. This finding, although subjective and based on common sense, nevertheless is important to the clinician.

\section{Discussion}

Previous studies on the use of chin cup appliances on human subjects have reported varying levels of success. ${ }^{8,9,15-19}$ Differences in these findings may be causally related to the variability of appliance design, duration of treatment, level of force utilized in the appliance, and/or the age of the subjects being treated. Experimental animal studies testing the effect of posteriorly directed forces against the temporomandibular joint have had more consistent results. Definite changes in mandibular development have been reported in these studies, which have used both radiographic and histologic techniques. ${ }^{9-14,30,31}$ The disparity between the results of human and experimental animal studies, added to the inconsistency of findings within the human experiments themselves, has produced uncertainty in the minds of orthodontic clinicians. ${ }^{32,33}$

In the present study, spceific evaluation has been made of fifty-seven hardand soft-tissue measurements in order to evaluate the efficacy of the orthopedic chin cup appliance. While the important individual facial component alterations have been discussed in the preceding section, a consideration of changes as they 


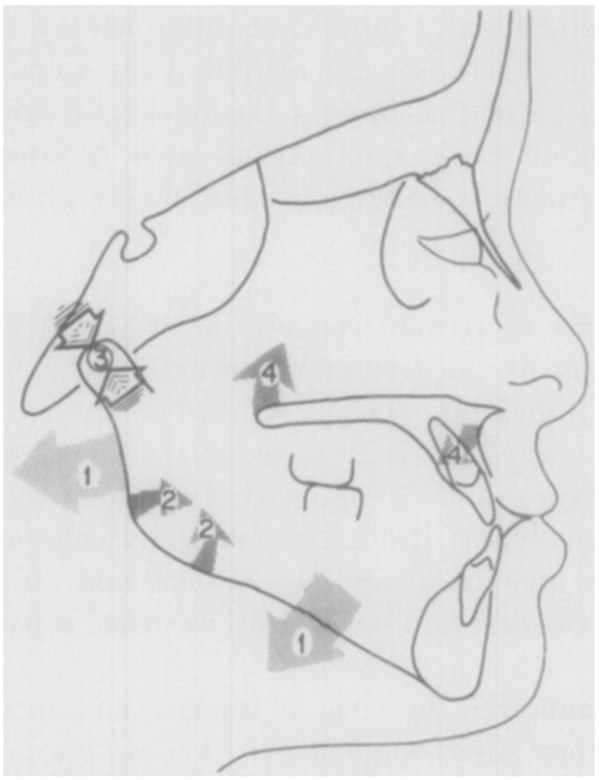

Fig. 6. Major contributions to correction of the Class III skeletal malocclusion. 1, The mandible rotated posteriorly, placing the ramus in a more vertical orientation to the cranial base; 2, the gonial angle was decreased, re-establishing the mandibular plane by overcoming changes introduced by posterior mandibular rotation; 3, vertical condylar growth was restricted; 4, the maxilla rotated slightly in a "clockwise" direction.

relate to the total facial pattern and its alteration are important for clinical evaluation and utilization of chin cup therapy.

Craniofacial component interaction. The differences between the treated and untreated subjects in size and position of the various facial components are small when taken as individual measures. The true clinical significance of their differences is seen only when the components of the craniofacial complex, having been dissected for ease of evaluation and discussion, are reassembled into their functional whole. Small differences in shape and orientation are additive and are, indeed, responsible for the over-all changes observed between the control and treatment groups.

The most significant craniofacial effects of the orthopedic chin eup forces appear to have been on the mandible and posterior face. The initial Angle Class III skeletal malocelusion was resolved by a combination of factors. First, posterior rotation of the mandible redirected the growth of the condyle into a more vertical vector (Fig. 6). This rotational effect was similar to that reported by both Armstrong ${ }^{15}$ and Suzuki. ${ }^{19}$ Second, there was a definite decrease in the gonial angle, which was greater than that seen in the untreated sample. Third, there was a significant decrease in the amount of vertical condylar growth, a finding which is consistent with earlier reports on experimental animals. ${ }^{9-14}$

There were concurrent decreases in the amount of growth in the posterior maxilla, partially matching a lack of vertical growth in the ramus. Indeed, as the 
mandible was rotated posteriorly, the maxilla, buffered by the intervening dentition and alveolar bone, rotated in the same direction. Therefore, while individual differences between the facial components measured on the linear or angular scale appeared small, the end result was a definite change in the structure of the mandible and maxilla. While orthopedic effects on the basicranium may have been relatively small, there can be no doubt as to the definite effects on the position and shape of the facial bones, particularly the mandible.

\section{Summary}

The purpose of this work was to provide a comprehensive description of craniofacial alteration in the Class III patient in response to orthopedic chin cup treatment. Thirty patients with skeletal Class III malocclusion under treatment with the chin cup appliance, averaging 6 years of age at the start of treatment, were followed longitudinally for a 3-year period. This treatment sample was compared cephalometrically with an analogous untreated Class III sample.

The following significant craniofacial alterations were noted in the sample that underwent orthopedic chin cup therapy:

1. A retardation of vertical ramus growth.

2. A retardation of vertical development in the posterior aspect of the mandibular body.

3. A retardation of vertical development in the posterior maxilla.

4. A closure of the gonial angle.

5. A distal rotation of the mandibular complex.

6. A decreased amount of anteroposterior anterior cranial base growth.

7. A redirection of the predominantly horizontal mandibular growth pattern to a more vertical dircetion.

8. A reduction of the maxillomandibular malrelationship toward normative values.

9. A production of an Angle Class I dental relationship following the establishment of normal maxillomandibular relations.

10. A lack of detectable localized effect on the symphyseal region or incisor position as a direct result of chin cup placement and pressure.

11. Development of soft-tissue profile changes in harmony with underlying skeletal changes.

While all of the listed alterations are important when analyzed separately, they gain increased importance when considered together. With orthopedic chin cup therapy, there is a change in craniofacial pattern leading to the observed resolution of the Angle skeletal Class III. malocclusion. This study thus provides strong support for the use of the orthopedic-force chin cup appliance in the clinical management of young patients with skeletal mandibular prognathism.

The author would like to thank the staff and faculty of the orthodontic departments at Northwestern University and the University of Chicago, as well as the Center for Human Growth and Development and the Biometries Laboratory, Sehool of Dentistry, University of Michigan, for their assistance in the preparation of this manuseript. 


\section{REFERENCES}

1. Hunter, J.: The natural history of the human teeth. Part II. A practical treatise on the disease of the teeth intended as a supplement to the national history of those parts, London, 1778, J. Johnson.

2. Goldstein, A.: Appraisal of results of surgical correction of Class III malocclusion, Angle Orthod. 17: 59-91, 1947.

3. Mills, L. F.: Epidemiologic studies of occlusion. IV. The prevalence of malocelusion in a population of 1,455 school children, J. Dent. Res. 45: 332-336, 1966.

4. Horowitz, S. L., Converse, J. M., and Gerstman, L. J.: Craniofacial relationships in mandibular prognathism, Arch. Oral Biol. 14: 121-131, 1969.

5. Thilander, B., and Myrberg, N.: The prevalence of malocelusion in Swedish schoolchildren, Scand. J. Dent. Res. 81: 12-20, 1973.

6. Weinberger, B. W.: Orthodontics: An historical review of its origin and evolution, St. Louis, 1926, The C. V. Mosby Company.

7. Graber, L. W.: Craniofacial alterations of human skeletal Class III malocclusion produced by chin cup-orthopedic force: A three-year longitudinal cephalometric study, Master's thesis, Northwestern University, 1973.

8. Graber, 'T. M., Chung, D. D. B., and Aoba, T. J.: Dentofacial orthopedics versus orthodontics, J. Am. Dent. Assoc, 75: 1145-1160, 1967.

9. Matsui, Y.: Effect of chin cap on the growing mandible, J. Jap. Orthod. Soc. 24: 165181, 1965.

10. Breitner, C.: Bone changes resulting from experimental orthodontic treatment, AM. J. ORTHOD. ORAL SURG. 26: 521-547, 1940.

11. Janzen, E. K., and Bluher, J. A.: The cephalometric, anatomic and histologic changes in Maoaca mulatta after application of a continuous-acting retraction force on the mandible, AM. J. ORTHOD. 51: 823-855, 1965.

12. Kulis, R. W.: A gross anatomic, cephalometric, and radiographic study of the influence of orthopedic forces on the vector of mandibular growth in Macaca mulatta, Master's thesis, Northwestern University, 1972.

13. Bare, M. R.: Influence of orthopedic forces on the vector and pattern of mandibular growth in Macaca mulatta monkeys: A histologie and gross anatomic study, Master's thesis, Northwestern University, 1972.

14. Petrovic, A. G., Stutzman, J. J., and Oudet, C. L.: Control processes in the postnatal growth of the condylar cartilage of the mandible. In McNamara, J. A., Jr. (editor): Determinants of mandibular form and growth, Monograph No. 4, Craniofacial Growth Series, Ann Arbor, 1975, Center for Human Growth and Development, pp. 101-154.

15. Armstrong, C. J.: A clinical evaluation of the chin cup, Aust. Dent. J. 6: 338-346, 1961.

16. Thilander, B.: Treatment of Angle Class III inalocclusion with chin cap, Trans. Eur. Orthod. Soc., pp. 384-398, 1963.

17. Thilander, B.: Chin-cap treatment for Angle Class III malocelusion: A longitudinal study, Trans. Eur. Orthod. Soc., pp. 311-327, 1965.

18. Irie, M., and Nakamura, S.: Orthopedic approach to severe skeletal Class III malocclusion, AM. J. ORTHOD. 67: 377-392, 1975.

19. Suzuki, N.: A cephalometric observation on the effect of the chin cap, J. Jap. Orthod. Soc. 31: 64-74, 1972.

20. Moyers, R. E.: Handbook of orthodontics, ed. 3, Chicago, 1973, Yearbook Medical Publishers.

21. Walker, G. F.: Personal communication.

22. Jacobson, A., Evans, W. G., Preston, C. B., and Sadowsky, P. L.: Mandibular prognathism, Aм. J. ORThOD. 66: 140-171, 1974.

23. Björk, A.: Variations in the growth pattern of the human mandible: Longitudinal radiographic study by the implant method, J. Dent. Res. 42: 400-411, 1963.

24. Joffe, B. M.: Cephalometric analysis of mandibular prognathism, J. Dent. Assoc. S. Afr. 20: 145-156, 173-180, 212-219, 1965. 
25. Rakosi, T.: The significance of roentgenographic cephalometries in the diagnosis and treatment of Class III malocclusions, Trans. Eur. Orthod. Soc., pp. 155-170, 1970.

26. Henry, H. L.: Craniofacial changes induced by "Orthopedic Forces" in the Macaca mulatta rhesus monkey, Master's thesis, University of Manitoba, 1973.

27. Jarabak, J. R.: University of Chicago cephalometric analysis, Orthodontic Seminar, University of Chicago, 1973.

28. Hirshfeld, W. J., and Moyers, R. E.: Prediction of craniofacial growth: The state of the art, AM. J. ORTHOD. 60: 435-444, 1971.

29. Hopkin, G. B.: The cranial base as an etiologic factor in malocelusion, Angle Orthod. 38: 250-255, 1968.

30. Adams, C. D.: The effect of continuous posterior mandibular forces (Class III) on the temporomandibular joint and dentofacial skeleton of Macaca mulatta, Master's thesis, University of Washington, 1969.

31. Joho, J. P.: The effects of extraoral low-pull traction to the mandibular dentition of Macaca mulatta, AM. J. ORTHOD. 64: 555-577, 1973.

32. Sassouni, V:. Dentofacial orthopedies: $\Lambda$ critical review, AM. J. Orthod. 61: 255-269, 1972 .

33. Cleall, J. F.: Dentofacial orthopedics, AM. J. ORTHOD. 66: 237-250, 1974.

1111 East Catherine St. (48109)

THE JOURNAL 60 YEARS AGO

July, 1917

"Bony tissue," says Oppenheim, "be it compact or cancellated, reacts to pressure by a transformation of its entire architecture; this takes place by resorption of the bone present and deposition of new bony tissue; both processes occur simultaneously. Deposition finally preponderates over resorption. The newly formed bone spicules are arranged in the direction of the pressure. Increased pull has similarly addition of new bony tissue as a result, and simultaneous orientation of the spicules thereof in the direction of the pull.

"The entire transformation of the architecture and the orientation of the newly formed spongy bone spicules, always occur so characteristically and lawfully, that we can say by the histological preparations in what manner the movements were accomplished. This characteristic transformation results only upon the application of very slight, physiologicallike influences. Should the force be too strong, the result will be such serious injuries to the periosteum, due to the disturbances in circulation that there will be no typical reaction of the bony cells." (Bernhard Wolf Weinberger: Orthodontia-lts purpose, problems, and possibilities, International Journal of Orthodontia, predecessor of the American Journal of Orthodontics, 3: 373-394, 1917.) 